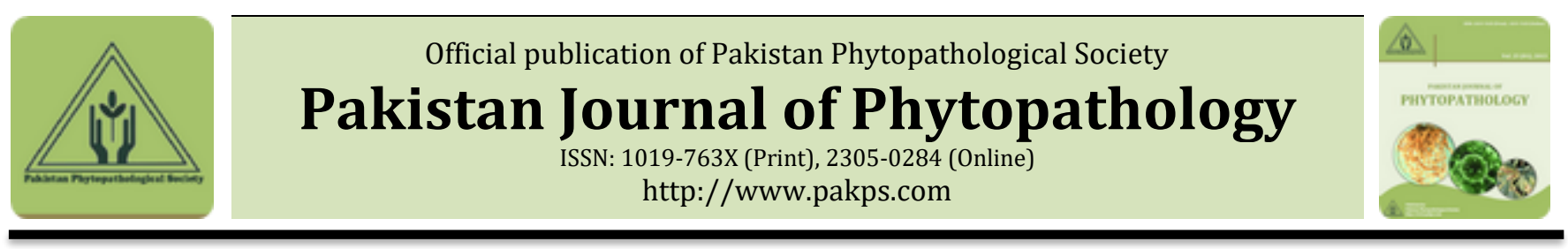

\title{
EFFICACY OF SACCHAROMYCES CEREVISIAE TO CONTROL CROWN ROT OF BANANA CAUSED BY FUSARIUM SEMITECTUM
}

\author{
aMuhammad Ather, aMuhammad Waris*, aMuhammad Azhar, aSaeed Ahmed, bMasood Ahmed, \\ aMuhammad Basharat, cMuhammad Mohsin \\ a Sindh Agriculture University, Tando Jam, Pakistan. \\ ${ }^{\boldsymbol{b}}$ Balochistan Agriculture College Quetta, Pakistan. \\ c Directorate of Soil Fertility, Agriculture Extension, Dhadhar, Pakistan.
}

\section{A B S T R A C T}

Crown rot disease of Banana is adversely affecting the quantity and quality of the fruits for consumption and utilization. The bananas collected from the local markets of Hyderabad, Tando Allahyar and Quetta were found suffered with different types of rots such as crown rot, anthracnose, fruit rot and stem end rot. Among them crown rot of banana was found predominantly followed by anthracnose and other fruit rots. Incidence and severity varied with the markets and locations. Fusarium semitectum found predominately associated with the affected fruits showing crown rot symptoms. So, yeast (Saccharomyces cerevisiae) were evaluated against F. semitectum under in-vitro conditions. S. cerevisiae also tested against crown rot disease development of banana fruit inoculated with $F$. semitectum. The addition of Saccharomyces cerevisiae in the culture medium has brought $93 \%$ reduction in the growth of $F$. semitectum. yeast was also tested against crown rot disease development on banana fruits by direct contact method. Yeast treatment brought more than $40 \%$ reduction in crown rot disease development as compared to the untreated fruits. The present study indicates that active yeast has the potential to manage harmful plant pathogens and thus could provide an alternate, reliable and eco-friendly control of crown rot disease.

Keywords: Saccharomyces Cerevisiae, Crown Rot, Banana, Fusarium semitectum.

\section{INTRODUCTION}

Banana is the fourth most important fruit crop of the world and delivers an important source of starch, particularly in Asia and Africa. It has appraised that plantain and other bananas supply more than 200 calories per day to 60 million people in Africa (Stover and Simmonds, 1987). In the world its production surpasses 91,000 thousand tons. India ranked on top in the world with the production of 27,575 thousand tons; while, Pakistan produces 115.5 thousand tons on 27238 hectares with an average yield of 424.23 (kg/ha) (FAOSTAT, 2013). In Pakistan, Sindh province is the

Submitted: February 12, 2018

Revised: May 28, 2018

Accepted for Publication: June 07, 2018

* Corresponding Author:

Email: waris.faqir@gmail.com

(C) 2017 Pak. J. Phytopathol. All rights reserved. main banana growing region where the soil and climatic conditions are satisfactory for its cultivation and it contribute $87 \%$ of the total country production (Ramma et al., 1999. Thatta, Hyderabad, Badin, Mirpurkhas, Tando Allahyar, Matiari, Tando Muhammad Khan, Sangar, Naushero Feroz, and Nawabshah are the main districts for banana production, However, its cultivation has extended to northern parts of Sindh particularly district Khairpur. It has been observed that green plantain and banana are not easily attacked by rot fungi even when they are in association with the fruit (Ramma et al., 1999). As ripening of sets starts, the activities of fungi even the saprophytic ones become more apparent on the fruit. The rate of ripen in plantain and banana varies as well as the rate of attack and destruction by the rot fungi also varies (Ogazi, 1996). Several fungal diseases reduce the quality and postharvest shelf life of this fruit crop (Win et al., 2007). The most common 
postharvest diseases of banana are stem end rot (Lasiodiplodia theobromae) (Rattanakreetakul, 2013) Fusarium semitectum, is recognized as the main fungi associated with crown rot of banana (Indrakeerthi and Adikaram, 2011). In some countries, crown rot disease is responsible of causing high losses; up to Eighty six percent (86\%) of bananas have been observed with crown rot symptoms during the rainy season having no chemical treatment after harvest. (Alvindia et al., 2000).

Extensive postharvest damage of the fruit can be caused by this disease and therefore, be deliberated as the large danger to native and export market of banana (Khan et al., 2001). Over $10 \%$ losses have been recorded in the United Kingdom from the Windward Islands during rainy season (Krauss and Johanson, 2000). Losses as high as $86 \%$ has also reported in untreated bananas from Philippines (Alvindia et al., 2000).

In commercial pack houses crown rot of banana usually managed with the treatment of systemic fungicides (Lassois et al., 2008). In spite of the yield losses caused by postharvest diseases, sufficient attention has not been given to research on this group of diseases (Yesuf et al., 2009). Emphasis must be given to explore risk of chemicals or alternative of chemical control methods be reduced hence, the present study was conducted to explore the potential of essential oils and yeast for the control of crown rot of banana with the following objectives:

To study the postharvest rots associated with banana.

To evaluate Saccharomyces cerevisiae for the control of Fusarium semitectum causing crown rot of banana under in-vitro conditions.

To evaluate Saccharomyces cerevisiae against crown rot disease development of banana fruit inoculated with Fusarium semitectum.

\section{MATERIALS AND METHODS}

Survey to record the incidences of postharvest diseases of banana: Local market of Hyderabad, Tando Allahyar and Quetta were visited to record the incidences of fruit rot of banana in the month of June and July 2016. The percent disease incidence of banana was calculated according to the following formula:

$$
\text { Disease incidence (\%) } \frac{\text { Number of diseased bananas }}{\text { Total number of bananas observed }} \times 100
$$

Banana fruits showing typical crown rot symptoms were collected from these markets and were brought to the laboratory of Plant Pathology Department, Balochistan Agriculture College Quetta for the isolation and identification of associated pathogens.

Isolation, purification and multiplication of disease causing organism: The rotted pieces of the crown region of banana were cutted into small pieces (three $\mathrm{mm}^{2}$ ) with the help of sterilized scissors. These pieces were surface sterilized with $5 \%$ commercial bleach (Sodium hypochlorite) for 3 minutes and placed in sterilized Petri dishes containing PDA medium amended with anti-bacterial to avoid bacterial contamination under aseptic condition. Five pieces were placed in each Petri dish. These Petri dishes were incubated at $28^{\circ} \mathrm{C}$ for 4 days. As far as, the fungal colonies were found over medium, these were sub cultured on another petri plate having PDA after observing the mycelia grown under microscope for further purification multiplication on PDA medium, through Hyphal Tip Culture.

The isolated fungi were identified on the basis of their morphological characteristics depending on colony structure, colony colour, shape and size of spores/conidia under microscope by using the key developed by Barnett and Hunter (1972). The pure culture was revised on new PDA plates after every month and used for the entire research work.

Pathogenicity test: Unripened banana of Bassrai variety was obtained. Banana hands were washed with water in order to remove the dust and mud. Bunches were separated from the banana hands and allowed 30 min to flow out the latex. Next the bunches were sterilized by spraying with $70 \%$ ethanol. Wait at least 30 min for the ethanol to dry and inoculated by placing the culture disks $(5 \mathrm{~mm})$ of $F$. semitectum. Disks were cut from the edges of actively growing culture on PDA medium and placed on top side of the crown. After inoculation the inoculated side and culture discs were covered with wet blotting paper to prevent the desiccation of the fungus and facilitate the easy penetration. Fruits were placed in racks, covered with polyethylene to avoid desiccation, and incubated at room temperature. After 12 hours of incubation wet blotting paper and culture disks were removed transferred to air conditioned room $\left(16^{\circ} \mathrm{C}\right)$ and $80 \%$ humidity for symptoms development. Observations were recorded on the basis of symptoms development and after 20 days of storage.

Effect of Saccharomyces cerevisiae on the linear growth of the Fusarium semitectum: Active 
Saccharomyces cerevisiae were tested against Fusarium semitectum under in-vitro conditions. Saccharomyces cerevisiae suspension was made by adding $1 \mathrm{~g}$ dried biomass in $5 \mathrm{ml}$ of water. One $\mathrm{ml}$ suspension of Saccharomyces cerevisiae pours on the surface of $20 \mathrm{ml}$ solidified medium in Petri dishes. Similarly $1 \mathrm{ml}$ of sterilized water was poured in control Petri dishes. The experiment was carried out with 3 independent replications. Whereas, $5 \mathrm{~mm}$ disk of Fusarium semitectum from the 8 days old culture was inoculated in each Petri dish. After inoculations these Petri plates were incubated and data was recorded as described above.

To evaluate Saccharomyces cerevisiae against crown rot disease development of banana fruit inoculated with Fusarium semitectum: The inoculated fruits were applied with yeast suspension by brush. Brush were dipped in Saccharomyces cerevisiae suspension (1 g dried biomass in $5 \mathrm{ml}$ of water) and applied to the crown area. The treated fruits were transferred to air conditioned room $\left(16^{\circ} \mathrm{C}\right)$ for 20 days. Inoculated fruits without the treatment of yeast served as control. Observations on symptoms development was recorded as described above.

\section{STATISTICAL ANALYSIS}

The collected data was analyzed statistically by employing the Fisher analysis of variance technique (Steel et al., 1997).

\section{RESULTS AND DISCUSSION}

Incidences of postharvest rots banana: Survey of local markets of Hyderabad, Tando Allahyar and Quetta reveled that crown rot of banana was found predominantly followed by anthracnose and other fruit rots. Incidence and severity varied among the markets and survey locations (Figure 1). The maximum disease incidences was recorded in Quetta market (50\%) followed by Hyderabad (42\%) and Tando Allahyar (37\%). Whereas, the individual incidences of crown rot of banana was 15, 30 and 27\% in Quetta, Hyderabad and Tando Allahyar, respectively.

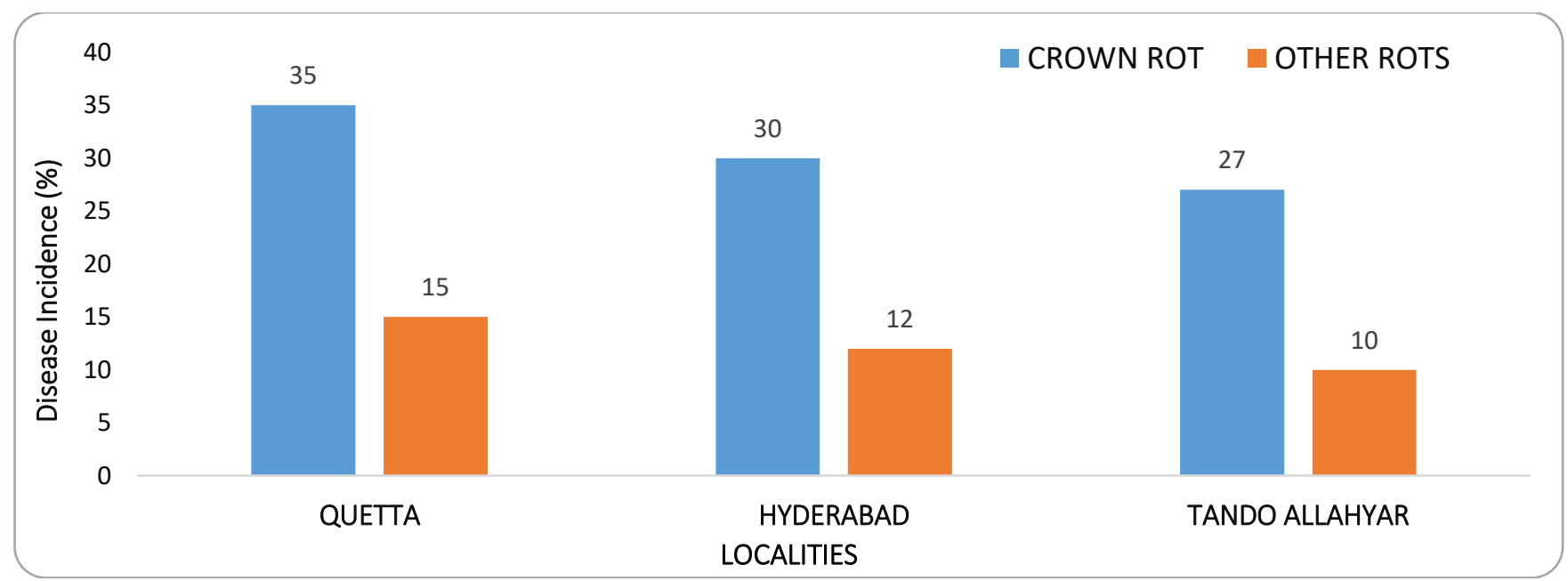

Figure 1. Incidences of postharvest rots of banana at different locations.


Figure 2. Postharvest rots associated with banana. 
Effect of Saccharomyces cerevisiae on the growth of $\boldsymbol{F}$. semitectum: The Use of Saccharomyces cerevisiae in the culture medium against $F$. semitectum has brought remarkable reduction in the growth of pathogen in all replication of treatment and the growth of targeted pathogen inhibited to 93\% when grown in Saccharomyces cerevisiae amended medium as compared to the control (un-amended medium) (Figure 3-4).



Figure 3. Effect of Saccharomyces cervisiae on linear colony growth of Fusarium semitectum. Each bar represents the mean of 3 replications.



Figure 4. Picture showing effect of Saccharomyces cervisiae on the growth of Fusarium semitectum. 
brought significant reduction in crown rot disease development as compared to the control (untreated fruits). (40.33\%) reduction in crown rot development found on fruits treated with Yeast. Similarly, significant minimum size disease lesions of $18.72 \mathrm{~cm}$ were recorded in fruits treated with yeast. (Figure 5-6).

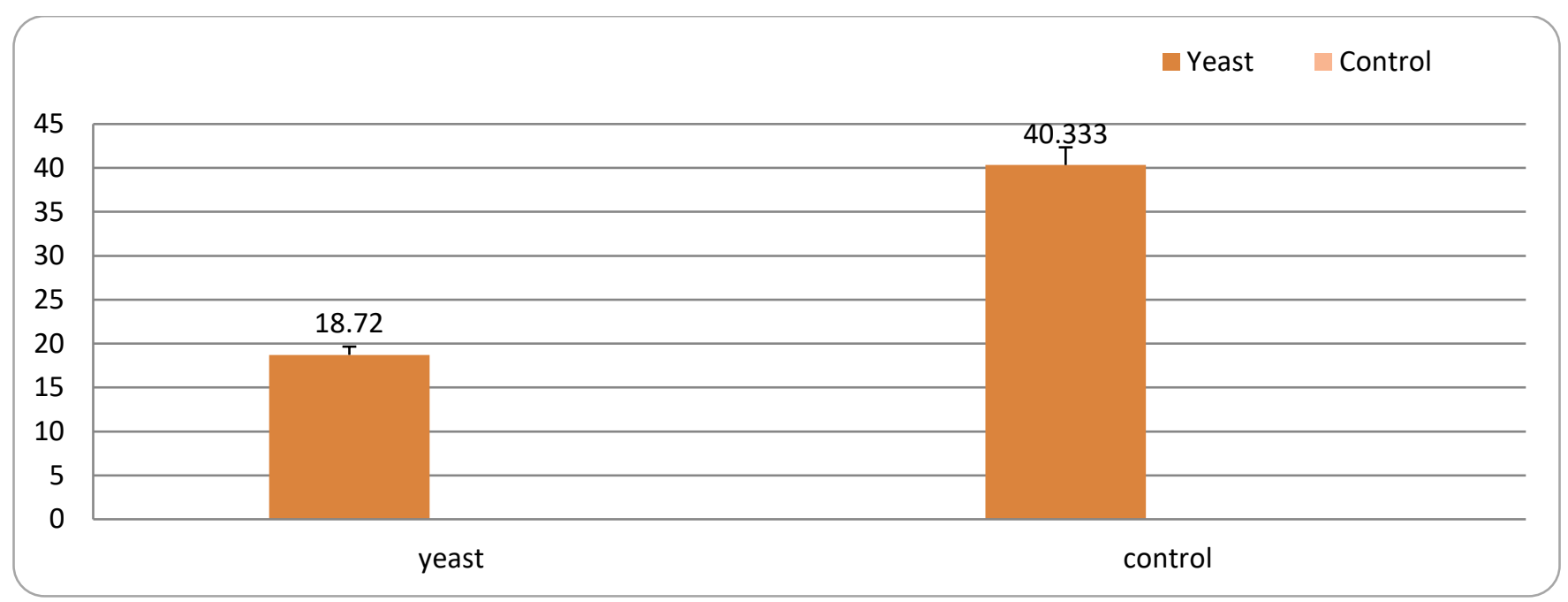

Figure 5. Effect of constitutive metabolites by yeast on crown rot disease development (lesion area) of banana fruits artificially inoculated by F. semitecum.

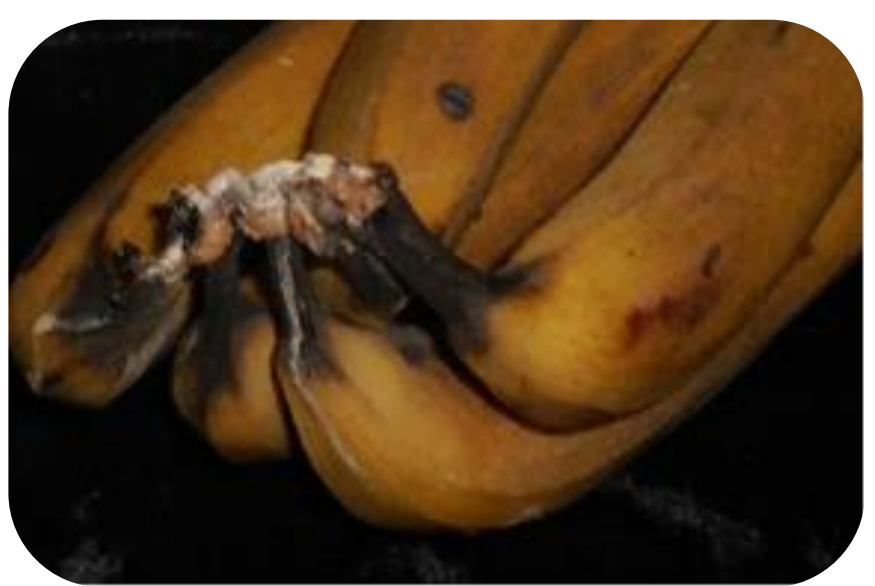

(A)

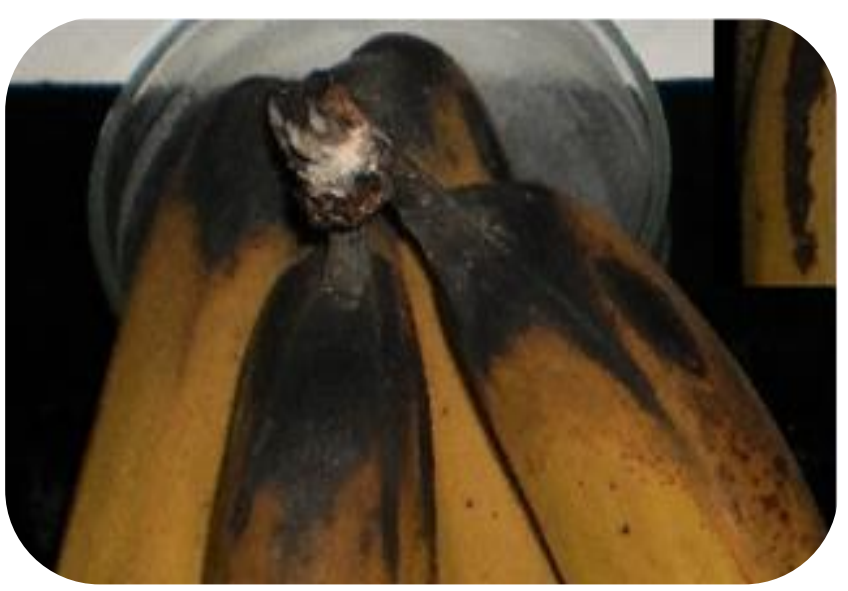

(B)

Figure 6. Picture showing effect of crown rot disease development of banana fruits artificially inoculated by $F$. semitecum. (A) yeast (Saccharomyces cervisiae) and (B). control.

\section{DISCUSSION}

Banana fruits of the local market of Hyderabad, Tando Allahyar and Quetta cities of Pakistan are found effected with varying incidence of different types of postharvest rots. Among the rots, crown rot of banana found to be the most severe disease and causing losses 25 to 35 percent. Similarly Krauss and Johanson, (2000), have reported over 10-86\% losses from UK and Philippines in bananas harvested during rainy periods. Rainy environment favors the disease development in Pakistan, UK and Philpines.
Alvindia et al., (2000) isolated Fusarium semitectum predominantly from the diseased tissues obtained from bananas showing crown rot symptoms. Furthermore Jimenez et al., (1993) also depicts the predominant association of this pathogen with banana crown rot. As the present study also shown the same result

Yeast has a promising history for the antimicrobial activity against a number of plant diseases. in present in vitro study Saccharomyces cerevisiae used against crown rot of banana pathogen and disease development with effective results of controlling disease up to 40 percent 
through direct contract meth. Simillarly Rinki and Tripathi et al., (2015) also found 100\% inhibition of fruit rotting fungus Colletotrichum musae causing anthracnose of banana and food by direct contact method through yeast for treatment of banana. And AbdAlla et al., (2014) recommended application of sweet almond oils in commercial pack houses as a safe and cost-effective management methods for the crown rot disease of banana. These results showing the same effect of essential oils as used in this research "Saccharomyces cerevisiae in the culture medium caused $93 \%$ reduction in the growth of $F$. semitectum due to its anti-fungal potential". Furthermore, Shalaby and El-Nady, (2008) found that the application of $S$. cerevisiae decrease the colony growth of $F$. oxysporum in culture media. After the observation of antagonistic effect of yeast to crown rot pathogen, it was applied to artificially inoculated bananas. Significant reduction in crown rot disease development as compared to the control (untreated fruits) was observed. Similarly EL-Mehalawy, (2004) explored the antagonistic potential of rhizosphere yeast fungi for the management of plant pathogenic fungi. Saccharomyces unispora were found having inhibitory effects on the growth of Fusarium oxysporum. Application of these two species in artificially infested soil with Fusarium oxysporum increased the kidney beans plant growth. Captioned results of scientists also reflects with this my research as these intimate about anti-fungal potential of essential oils against different pathogens same used in this research against pathogen.

\section{CONCLUSION}

The present studies revealed that banana fruits were severely affected by various postharvest rots. Crown rot of banana caused by F. semitectum was the predominant postharvest rots, widely prevailed in the study areas. The yeast (Saccharomyces cerevisiae) was found significantly inhibited the colony growth of $F$. semitectum in vitro conditions.

\section{RECOMMENDATION}

In order to explore the alternative of chemical control methods, different strategies have been tried time to time. Yeast has a promising history for the antimicrobial activity against a number of plant diseases. The present studies indicate that active yeast, have the potential to control harmful plant pathogens and thus could provide an alternate, reliable and ecofriendly control mechanism. Therefore, more comprehensive studies should be carried out in order to determine its effects on other plant pathogens causing destructive diseases in crop plant.

\section{REFERENCES}

Abd-Alla, M. A. 2014. Post-harvest treatments for controlling crown rot disease of Williams banana fruits (Musa acuminata L.) in Egypt. Plant Pathology \& Quarantine, 4: 1-12.

Alvindia, D. G., T. Kobayashi, Y. Yaguchi and K. T. Natsuaki. 2000. Symptoms and the associated fungi of postharvest diseases on non-chemical bananas imported from the Philippines. Japanese Journal of Tropical Agriculture, 44: 87-93.

Barnett, H.L and B.B Hunter. 1972. Illustrated genera of imperfect fungi. $3^{\text {rd }}$ edition Burgess Publishing Co., pp. 273.

Barnett, K. R. P., and B. B. Hunter. 1972. Illustrated Genera of Imperfect Fungi. Mycologia, 64: 930.

El-Mehalawy, A. 2004. The rhizosphere yeast fungi as biocontrol agents for wilt disease of kidney bean caused by Fusarium oxysporum. International Journal of Agriculture and Biology (Pakistan).

FAOSTAT. 2013. Food and agriculture organization, statistical report 2013. http://faostat3.fao.org.

Heinisch, O. 1962. Steel, R. G. D., and J. H. Torrie: Principles and Procedures of Statistics. (With special Reference to the Biological Sciences.) McGraw-Hill Book Company, New York, Toronto, London 1960, 481 S., 15 Abb.; 81 s 6 d. Biometrische Zeitschrift, 4: 207-208.

Indrakeerthi, S. R. P. and N. K. B. Adikaram. 2011. Control of crown rot of banana using Carica papaya latex. Journal of the National Science Foundation of Sri Lanka, 39: 155.

Jiménez, M., A. Logrieco and A. Bottalico. 1993. Occurrence and Pathogenicity of Fusarium Species in Banana Fruits. Journal of Phytopathology, 137: 214-220.

Khan, S. H., J. Aked and N. Magan. 2001. Control of the anthracnose pathogen of banana (Colletotrichum musae) using antioxidants alone and in combination with thiabendazole or imazalil. Plant Pathology, 50: 601-608.

Krauss, U. and A. Johanson. 2000. Recent advances in the control of crown rot of banana in the Windward Islands. Crop Protection, 19: 151-159.

Lassois, L., L. de Lapeyre de Bellaire and M. H. Jijakli. 2008. Biological control of crown rot of bananas with Pichia anomala strain $\mathrm{K}$ and Candida oleophila 
strain O. Biological Control, 45: 410-418.

Ogazi, P. 0. 1996. Plantain: Production, Processing, Utilisation. Paman and Associates.

Ramma, S.P., B. Madhu and P. Peerthum. 1999. Postharvest quality improvement of banana. Food and Agricultural Research Council: 187-194.

Rattanakreetakul, C. 2013. Fumigation with plant volatile oils to control stem end rot of banana. Acta Horticulturae: 207-213.

Shalaby, M. and M. F. El-Nady. 2008. Application of Saccharomyces cerevisiae as a biocontrol agent against Fusarium infection of sugar beet plants. Acta Biologica Szegediensis, 52: 271-275.

Singh, R. and P. Tripathi. 2015. Cinnamomum zeylanicum essential oil in the management of anthracnose of banana fruits. Journal of Innovations in Pharmaceutical and Biological Sciences, 2: 290-299.

Steel, R.G.D., J.H. Torrie and D.A. Dicky. 1997. Principles and Procedures of Statistics: A Biometric approach. 3rd Ed. McGraw Hill Book Co., New York.

Stover, R. and N. Simmonds. 1987. Bananas. Tropical agricultural series. John Wiley and Sons, Inc., NY, 10: $158-468$.

Tripathi, J. N., R. O. Oduor and L. Tripathi. 2015. A highthroughput regeneration and transformation platform for production of genetically modified banana. Frontiers in plant science, 6: 1025.

Win, N. K. K., P. Jitareerat, S. Kanlayanarat and S. Sangchote. 2007. Effects of cinnamon extract, chitosan coating, hot water treatment and their combinations on crown rot disease and quality of banana fruit. Postharvest Biology and Technology, 45: 333-340.

Yesuf, M., W. Mandefro, E. Ahmed, G. Adugna, D. Tadesse, T. Hussien and M. Shehabu. 2009. Review of research on fruit crop diseases in Ethiopia. Increasing crop production through improved plant protection, 2: 231-251. 